\title{
Características maternas e dos recém-nascidos admitidos em uma unidade de terapia intensiva neonatal
}

\author{
Maternal and newborn characteristics admitted in an neonatal \\ intensive care unit
}

\begin{abstract}
Brenda Araújo Lopes' • Ana Lívia Castelo Branco de Oliveira ${ }^{2} \bullet$ Girlene Ribeiro da Costa ${ }^{3} \bullet$ Alessandra de

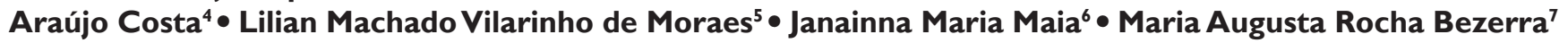

\begin{abstract}
RESUMO
Objetivo:Avaliar as características maternas e dos recém-nascidos admitidos em uma Unidade de Terapia Intensiva Neonatal pública. Método:Trata-se de um estudo do tipo retrospectivo, documental e quantitativo.Amostra censitária com I 25 prontuários de recém-nascidos admitidos em uma unidade de terapia intensiva neonatal de um hospital regional no Piauí, entre maio de 2017 e março de 2018. Os dados foram coletados utilizando formulário estruturado e analisados no Statistical Package for the Social Sciences 23.0.A pesquisa teve consentimento ético, parecer n. 2.8I7.447. Resultados:A maioria das mães dos neonatos tinham entre 20 e 34 anos, viviam em união estável, possuíam ensino médio completo, eram donas de casa e procedentes de municípios circunvizinhos à Floriano.Tiveram gestação única, com mais de cinco consultas de pré-natal, e idade gestacional de até 36 semanas. O percentual de cesáreas se igualou ao de partos normais. Os neonatos em sua maioria, eram do sexo masculino, pardos, com peso adequado ao nascer. Percentual importante dos RN necessitou de reanimação cardiopulmonar e oxigenoterapia. O diagnóstico de admissão mais prevalente foi a Síndrome do Desconforto Respiratório, seguido pela prematuridade. Conclusão: Os neonatos apresentaram variáveis de risco de morbibortalidade, o que sucinta condições de alerta para o monitoramento de agravos e direciona ações voltadas à prevenção de riscos à saúde deste grupo etário. Palavras-chave:Assistência à saúde; Unidades de Terapia Intensiva Neonatal; Enfermagem
\end{abstract}

\begin{abstract}
Objective:To evaluate the characteristics of mothers and newborns admitted to a public Neonatal Intensive Care Unit. Method:This is a retrospective, documentary and quantitative study. Census sample with 125 medical records of newborns admitted to a neonatal intensive care unit of a regional hospital in Piauí, between may 2017 and march 2018. Data were collected using a structured form and analyzed in the Statistical Package for the Social Sciences 23.0. The research had ethical consent, opinion no. 2,817,447. Results: Most mothers of newborns were between 20 and 34 years old, lived in a stable relationship, had completed high school, were housewives and came from municipalities surrounding Floriano. They had a single pregnancy, with more than five prenatal consultations, and a gestational age of up to 36 weeks. The percentage of cesarean sections was equal to that of normal births. Most newborns were male, brown, with adequate birth weight. A significant percentage of newborns required cardiopulmonary resuscitation and oxygen therapy. The most prevalent admission diagnosis was Respiratory Discomfort Syndrome, followed by prematurity. Conclusion:The neonates presented risk variables of morbidity and mortality, which succinctly alert conditions for the monitoring of diseases and direct actions aimed at the prevention of health risks in this age group.
\end{abstract}

Key-words: Delivery of Health Care; Intensive Care Units, Neonatal; Nursing.

NOTA

I Enfermeira. Graduada pela Universidade Federal do Piauí, Campus Amílcar Ferreira Sobral (Floriano, Piauí, Brasil). E-mail: brendaaraujo I205@gmail.com

2 Doutoranda em Enfermagem pelo Programa de Pós-Graduação em Enfermagem da Universidade Federal do Piauí (Teresina, Piauí, Brasil).E-mail: analiviacbranco@ hotmail.com

3 Doutoranda em Enfermagem pelo Programa de Pós-Graduação em Enfermagem da Universidade Federal do Piauí (Teresina, Piauí,Brasil).E-mail:gigiribeirocosta@hotmail.com 4 Especialista em Enfermagem Cardiovascular e Intervencionista pela Universidade Estadual do Ceará (UECE).(Teresina, Piauí,Brasil).E-mail:alessandra.araujoglu@gmail.com

5 Doutora em Saúde Coletiva pelo Programa de Pós-Graduação em Saúde Coletiva da Universidade Federal do Maranhão (São Luís, Maranhão, Brasil). Professora Adjunta I do Curso de Bacharelado em Enfermagem da Universidade Federal do Piauí, Campus Amílcar Ferreira Sobral (Floriano, Piauí, Brasil) E-mail: lilianvilarinho@hotmail.com

6 Enfermeira, bacharel pelo Centro universitário Santo Agostinho (Teresina, PI, Brasil).e-mail:janainnamaia@hotmail.com

7 Doutora em Enfermagem pela Universidade Federal do Piauí (2015-2018). Professora Adjunta Nível 2 da Universidade Federal do Piauí/CAFS, no Curso de Bacharelado em Enfermagem. E-mail: mariaaugusta@ufpi.edu.br. 


\section{INTRODUÇÃO}

O período neonatal, os primeiros 28 dias de vida do recém-nascido $(\mathrm{RN})$, é considerado o mais crítico para a saúde do indivíduo, com necessidade de cuidados intensivos visto que o neonato pode apresentar risco de vida em decorrência de baixo peso ao nascer (BPN) ou de algum processo patológico como anomalia congênita ${ }^{(1,2)}$.

Nesse contexto, a assistência de enfermagem neonatal vem avançando nos últimos anos, sendo a terapia intensiva um espaço de novas tecnologias em saúde. Nesses espaços emerge a necessidade de aprimoramento do conhecimento científico no que diz respeito à saúde e ao desenvolvimento do neonato, e sobre suas necessida$\operatorname{des}^{(3)}$.Ainda assim, a evolução tecnológica é insipiente na redução de morbimortalidade em neonatos.

A literatura internacional aponta para índices alarmantes de óbitos nos primeiros 28 dias de vida, principalmente em países e regiões mais pobres ${ }^{(4)}$. No Brasil, a mortalidade neonatal é $70 \%$ das mortes no primeiro ano de vida. No ano de 2016, as complicações do nascimento prematuro e durante $\circ$ trabalho de parto foram as causas de $30 \%$ das mortes de neonatos, evitáveis em sua maioria ${ }^{(4)}$. Isso alavanca o cuidado de enfermagem como instrumento mitigar o fenômeno.

Embora esse indicador de saúde apresente um perfil nacional de caráter decrescente, ele ainda persiste como um problema de saúde pública no Brasil, em virtude das desigualdades urbanas e das diferenças inter-regionais 0 que evidencia a necessidade de ampliação e melhoria das políticas públicas voltadas para assistência materno-infantil.

A Unidade de Tratamento Intensivo (UTI) neonatal é um serviço destinado ao cuidado integral de prematuros e RN com problemas ao nascer. Assim, entende-se que os cuidados intensivos nos primeiros dias de vida são essenciais para a redução da mortalidade neonatal. Entretanto, é preciso considerar as demais variáveis que podem estar associadas à morbidade neonatal tais como as condições socioeconômicas, escolaridade materna, assistência pré-natal e ao parto, dentre outras ${ }^{(5)}$.

Além disso há desigualdades na distribuição dos leitos e na qualidade da assistência prestada, sendo difícil prever fidedignamente os custos econômicos advindos disto(5). Logo as intervenções em saúde devem permear estas variáveis no sentido de prevenir agravos.

Apesar de um número significativo de estudos brasileiros a cerca do perfil de neonatos admitidos em UTI ${ }^{(2,6,7)}$, pouco se sabe da realidade no estado do Piauí, demostrando assim, a necessidade de pesquisas nesta direção. Faz-se necessário conhecer variáveis relacionadas a mãe e ao neonato que influenciem nos contextos de morbimortalidade dentro da UTI neonatal. Isto possibilita o nortear de ações e cuidados a este público, repercutindo na resolutividade clínica nas UTIs.
O contexto apresentado leva ao questionamento de pesquisa sobre como se comportam as características maternas e dos recém-nascidos admitidos na UTI neonatal de um hospital regional do Piauí. Portanto, definiu-se como objetivo do estudo avaliar as características maternas e dos recém-nascidos admitidos na UTI neonatal de um hospital regional do Piauí.

\section{MÉTODO}

Estudo descritivo, documental e retrospectivo realizado a partir de dados secundários oriundos de prontuários de pacientes admitidos em na UTI neonatal de um Hospital Regional localizado em Floriano, Piauí. Os prontuários foram acessados manualmente por meio do Serviço de Arquivamento Médico e Estatístico (SAME) do hospital, que é referência em atenção emergencial, com os serviços de UTI neonatal e adulta.

A população inicial do estudo foi composta por todos os neonatos admitidos na referida UTI neonatal desde a sua fundação em maio de 2017 até o momento da coleta de dados março de 2018, o que representou uma amostra censitária de 176 internações, dispensando cálculo amostral. No entanto foram disponibilizados para a pesquisa apenas os prontuários de pacientes que já tiveram o tratamento finalizado ou desfecho clínico, uma amostra de 125 prontuários.

Os participantes de pesquisa foram os recém-nascidos admitidos vivos na UTI Neonatal entre o período estabelecido; oriundos das salas de parto do próprio hospital ou de outras instituições de saúde. Os neonatos com mais de uma admissão na unidade foram incluídos na pesquisa considerando-se a primeira internação.

Excluíram-se neonatos com peso inferior a 500 gramas e/ou idade gestacional inferior a 24 semanas, uma vez que esses dados são considerados como aborto(8). Também foram excluídos pacientes portadores de anomalias congênitas incompatíveis com a vida, visto que não tiveram a oportunidade de intervenção obstétrica e/ou neonatal. Os prontuários cujos dados estavam incompletos, foram excluídos.

A coleta de dados ocorreu no período de outubro a novembro de 2018 e foi realizada por acadêmicos de enfermagem da Universidade Federal do Piauí (UFPI), devidamente treinados. Utilizou-se um formulário elaborado pelas próprias pesquisadoras e adaptado com base nas informações contidas nos prontuários. Realizou-se, um pré-teste, para fins de adequação do instrumento e facilitando a operatividade durante a coleta.

O instrumento continha variáveis sociodemográficas e as características maternas (idade, estado civil, escolaridade, procedência); obstétricas ( número de consulta de pré-natal, tipo de parto); e variáveis referentes ao $\mathrm{RN}$ (sexo, idade em dias, peso de nascimento, escore de Ap- 
gar no $1^{\circ}$ e $5^{\circ}$ minutos de vida), relacionadas a internação (diagnóstico de entrada na UTI, tempo de permanência na unidade, oxigênio suplementar) e ao desfecho clínico (alta, óbito).

A coleta de dados ocorreu após a concessão do Termo de Anuência pelo Núcleo de Educação Permanente do HRTN e mediante entrega do Termo de Compromisso de Utilização de Dados (TCUD), visando assegurar a confidencialidade das informações. Os dados foram digitados e armazenados em planilhas eletrônicas por meio do Mi-

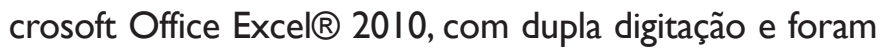
processados no Statistical Package for the Social Sciences (SPSS) for Windows, versão 23.0. Realizaram-se análises descritivas (medidas de tendência central) das variáveis.

O estudo foi aprovado pelo Comitê de Ética em Pesquisa (CEP) da UFPI, parecer $n^{\circ} 2.817 .447$ e cumpriu as determinações do Conselho Nacional de Saúde $n^{\circ} 510 / 2016$ que versa sobre procedimentos éticos adotados na pesquisa, agindo de forma a preservar a ética na pesquisa com seres humanos, assim como, respeitando a dignidade humana e a proteção dos participantes ${ }^{(9)}$.

\section{RESULTADOS}

Foram analisados 125 prontuários, através dos quais evidenciou-se que, a maioria das mães dos pacientes internados na UTI neonatal estavam em idade fértil reprodutiva (entre 20 e 34 anos). Entretanto, um percentual representativo estava nos extremos da idade, adolescentes ou acima dos 35 anos, cerca de $25 \%$. A maioria das mães viviam em união estável $(27,2 \%)$, se autodeclara- ram pardas $(27,2 \%)$, possuíam ensino médio completo (26,4\%), eram donas de casa $(22,4 \%)$, e procedentes de outros municípios do Piauí (74,4\%) (Tabela I).

Em relação às características relacionadas a gestação, ao pré-natal e ao parto verificou-se que a maioria das mães de bebês internados na UTI tiveram gestação única $(66,4 \%)$ e realizaram mais de cinco consultas de pré-natal (54,4\%). Em relação à Idade Gestacional (IG) observouse que $52,8 \%$ dos partos foram pré-termos e o percentual de cesáreas se igualou ao de partos normais $(49,4 \%)$ (Tabela 2).

A Tabela 3 mostra que os neonatos internados são em sua maioria do sexo masculino $(53,7 \%)$, de cor parda (36,8\%), com peso ao nascer de 2.500 gramas ou mais $(43,2 \%)$. Entretanto, chama a atenção o percentual de baixo peso ao nascer (37,6\%).

Em relação ao índice de Apgar no $1^{\circ}$ e $5^{\circ}$ minutos de vida, verificou-se que $35,2 \%$ dos bebês obtiveram o índice de 4 a 7, e 20,8\% deles obtiveram escore de 0 a 3 no $1^{\circ}$ minuto de vida, o que indica que não tiveram boa vitalidade ao nascer. No $5^{\circ}$ minuto, $40,8 \%$ dos bebês apresentaram boa vitalidade, entretanto, número expressivo dos bebês apresentou o índice entre 4 e 7 (28,0\%) e houve reanimação cardiopulmonar em uma quantidade importante dos casos (30,4\%) (Tabela 3).

A maioria dos neonatos foram admitidos na UTI neonatal devido ao desconforto respiratório (36,0\%), seguidos pela prematuridade $(14,4 \%)$, asfixia ao nascer $(9,6 \%)$ e aspiração de mecônio (6,4\%). Chama a atenção para $48,0 \%$ dos neonatos que necessitaram de oxigenoterapia

TABELA 1 - Caracterização socioeconômica e demográfica das mães dos neonatos internados na UTI neonatal, Floriano, Piauí, Brasil, 2017-2018.

\begin{tabular}{|c|c|c|c|c|c|}
\hline Variável & $\mathbf{N}$ & $\%$ & Variável & $\mathbf{N}$ & $\%$ \\
\hline Idade (em anos) & & & Ocupação materna * & & \\
\hline $14-19$ & 32 & 25,6 & Dona de casa & 28 & 22,4 \\
\hline $20-34$ & 62 & 42,6 & Lavradora & 22 & 17,6 \\
\hline 35 ou mais & 31 & 24,8 & Estudante & 13 & 10,4 \\
\hline Estado civil & & & Outros & 4 & 3,2 \\
\hline Solteira & 19 & 15,2 & Não registrado & 58 & 46,4 \\
\hline Casada & 12 & 9,6 & Grau de instrução & & \\
\hline União estável & 34 & 27,2 & Fundamental completo & 06 & 4,8 \\
\hline Não registrado & 60 & 48,0 & Fundamental incompleto & 21 & 16,8 \\
\hline Raça/ Cor & & & Médio completo & 33 & 26,4 \\
\hline Parda & 34 & 27,2 & Médio incompleto & 08 & 6,4 \\
\hline Não registrado & 91 & 72,8 & Superior completo & 03 & 2,4 \\
\hline Procedência & & & Superior incompleto & 02 & 1,6 \\
\hline Floriano- PI & 13 & 10,4 & Não registrado & 52 & 41,6 \\
\hline Outros no PI & 93 & 74,4 & - & - & - \\
\hline Outros estados & 12 & 9,6 & - & - & - \\
\hline Total & 125 & 100 & Total & 125 & 100 \\
\hline
\end{tabular}

*Ocupação: ato ou efeito de se ocupar; profissão; emprego; trabalho.

Fonte: prontuários. 
TABELA 2 - Dados relacionados à pré-natal e ao parto de mães de RN internados na UTI, Floriano,

Piauí, Brasil, 2017-2018.

\begin{tabular}{|c|c|c|c|c|c|}
\hline Variável & $\mathbf{N}$ & $\%$ & Variável & $\mathbf{N}$ & $\%$ \\
\hline \multicolumn{3}{|c|}{ Número de consultas de Pré-natal } & \multicolumn{3}{|c|}{ Idade Gestacional (em semanas) } \\
\hline Até 5 & 26 & 53,7 & Até 36 & 66 & 52,8 \\
\hline Mais de 5 & 68 & 43,3 & 37 a 41 & 43 & 34,4 \\
\hline Não registrado & 31 & 2,4 & 42 ou mais & 01 & 0,8 \\
\hline \multicolumn{2}{|c|}{ Tipo de Gestação } & & Não registrado & 15 & 15 \\
\hline Gemelar & 12 & 3,2 & Tipo de Parto & & \\
\hline Única & 83 & 1,6 & Cesárea & 58 & 49,4 \\
\hline Não registrado & 30 & 36,8 & Normal & 58 & 49,4 \\
\hline- & - & - & Não registrado & 09 & 7,2 \\
\hline Total & 125 & 100 & Total & 125 & 100 \\
\hline
\end{tabular}

Fonte: prontuários

TABELA 3 - Caracterização dos neonatos internados na UTI, Floriano, Piauí, Brasil, 2017- 2018.

\begin{tabular}{|c|c|c|c|c|c|}
\hline Variável & $\mathbf{N}$ & $\%$ & Variável & $\mathbf{N}$ & $\%$ \\
\hline Sexo & & & Apgar $1^{\circ} \mathrm{min}$ & & \\
\hline Masculino & 76 & 53,7 & $8-10$ & 22 & 17,6 \\
\hline Feminino & 46 & 43,3 & $4-7$ & 44 & 35,2 \\
\hline Não informado & 03 & 2,4 & $0-3$ & 26 & 20,8 \\
\hline Cor/ Raça & & & Não registrado & 33 & 26,4 \\
\hline Branca & 04 & 3,2 & Apgar $5^{\circ} \mathrm{min}$ & & \\
\hline Negra & 02 & 1,6 & $8-10$ & 51 & 40,8 \\
\hline Parda & 46 & 36,8 & $4-7$ & 35 & 28,0 \\
\hline Não informado & 73 & 58,4 & $0-3$ & 06 & 4,8 \\
\hline Peso ao nascer* & & & Não registrado & 33 & 26,4 \\
\hline$\geq 2.500 \mathrm{~g}$ ou mais & 54 & 43,2 & \multicolumn{3}{|c|}{ Reanimação RN em sala de parto } \\
\hline $1.500 \mathrm{~g}$ a $2.499 \mathrm{~g}$ & 47 & 37,6 & Sim & 38 & 20,0 \\
\hline $1.000 \mathrm{~g}$ a $1.499 \mathrm{~g}$ & 14 & 11,2 & Não & 25 & 30,4 \\
\hline Até $999 \mathrm{~g}$ & 09 & 7,2 & Não registrado & 62 & 49,6 \\
\hline Não informado & 01 & 0,8 & - & - & - \\
\hline Total & 125 & 100 & Total & 125 & 100 \\
\hline
\end{tabular}

Fonte: prontuários.

e também para a falta de registro para esta variável de 42,6\% (Tabela 4).

Observou-se que $20,8 \%$ estiveram internados por oito dias, seguidos dos que estiveram internados por até 7 dias (20\%). O tempo médio de dias de internação foi de 38,7 dias, com desvio padrão de 37,8 dias. O tempo mínimo de internação foi de I dia e o tempo máximo foi de 74 dias. Quanto aos desfechos dos neonatos internados na UTI, observou-se que a maioria deles receberam alta hospitalar $(69,6 \%)$, mas é válido destacar o percentual de óbitos de 16,8\% (Tabela 4).

TABELA 4 - Variáveis relacionadas a internação, a assistência e aos desfechos clínicos dos neonatos na UTI, Floriano, Piauí, Brasil 2017-2018.

\begin{tabular}{|c|c|c|c|c|c|}
\hline Variável & $\mathbf{N}$ & $\%$ & Variável & $\mathbf{N}$ & $\%$ \\
\hline \multicolumn{2}{|c|}{ Diagnóstico de Admissão } & & \multicolumn{3}{|c|}{ Tempo de permanência na UTIN**(dias) } \\
\hline Taquipnéia transitória & 07 & 5,6 & Até 7 dias & 25 & 20,0 \\
\hline Baixo peso ao nascer & 03 & 2,4 & $8-14$ dias & 26 & 20,8 \\
\hline Prematuridade & 18 & 14,4 & $15-30$ dias & 23 & 18,4 \\
\hline Sepse neonatal & 05 & 4,0 & $31-45$ dias & 11 & 8,8 \\
\hline SDR $^{*}$ & 45 & 36,0 & $46-50$ dias & 04 & 3,2 \\
\hline Asfixia ao nascer & 12 & 9,6 & $51-60$ dias & 03 & 2,4 \\
\hline Icterícia neonatal & 05 & 4,0 & 61 ou mais & 01 & 0,8 \\
\hline Aspiração de mecônio & 08 & 6,4 & Não registrado & 32 & 25,6 \\
\hline Pneumonia & 04 & 3,2 & \multicolumn{2}{|c|}{ Desfechos clínicos } & \\
\hline Infecção Neonatal & 03 & 2,4 & Alta & 87 & 69,6 \\
\hline Insuficiência cardíaca & 04 & 3,2 & Óbito & 21 & 16,8 \\
\hline Outros & 10 & 8,0 & Transferência & 05 & 4,0 \\
\hline Não registrado & 01 & 0,8 & Não registrado & 12 & 9,6 \\
\hline Oxigenoterapia & & & Oxigenoterapia & & \\
\hline Sim & 60 & 48,0 & Não & 13 & 10,4 \\
\hline Não registrado & 52 & 41,6 & - & - & - \\
\hline Total & 125 & 100 & Total & 125 & 100 \\
\hline
\end{tabular}

$\mathrm{SDR}^{\star}$ (Síndrome do Desconforto Respiratório); *UTIN: Unidade de Terapia Intensiva Neonatal.

Fonte: prontuários. 


\section{DISCUSSÃO}

Os resultados desse estudo puderam evidenciar o acesso aos serviços de saúde em busca de um leito de terapia intensiva neonatal, enquanto realidade no Piauí. Percebeu-se, contudo, $\mathrm{o}$ alto índice de cesáreas e partos pré-termos verificados e as demandas em saúde nos municípios circunvizinhos a Floriano, os quais não contam com serviços de saúde especializados, gerando um deslocamento geográfico das gestantes em busca dessa assistência neonatal intensiva.

Investigação conduzida pela Sociedade Brasileira de Pediatria (SBP) revelou que há carência de leitos em UTI neonatal, sendo mais distribuídos nas capitais e metrópoles. Tal investigação mostra que há desigualdade na distribuição das UTI Neonatais no país ${ }^{(10)}$. Ademais, a ausência de um aparato tecnológico, de recursos físicos e humanos necessários para a prestação de uma assistência de alta complexidade nos municípios menores interferem indiretamente na morbimortalidade mãe e neonato gravemente enfermo.

Das mães cujos bebês precisaram de cuidado intensivo, a maioria era dona de casa, com ensino médio completo.Além disso, parte significativa estava em idade fértil. Tais resultados corroboram com o grupo de mães estudadas em UTIs neonatais no Distrito Federal e outras cidades do Nordeste brasileiro(II).

Outra característica materna verificada neste estudo foi o grau de escolaridade das mães dos neonatos admitidos. Predominou dentre elas o ensino médio completo, sendo este dado considerado positivo, visto que estudiosos tem demonstrado associação entre filhos nascidos de mães com baixa escolaridade e morbimortalidade em UTI ${ }^{(12)}$.

A literatura diz que a melhor idade materna do ponto de vista reprodutivo está compreendida entre 20 e 25 anos, chamada de adulto jovem, período considerado de menor risco perinatal(6). Contudo, neste estudo haviam mães nos extremos da idade (adolescentes ou acima dos 35 anos), o que impõe risco ao nascimento e vitalidade do neonato.

Os extremos de idade são fatores de risco gestacional e piores condições perinatais, como por exemplo, o parto pré-termo, baixo peso ao nascer (BPN), e, portanto, maior necessidade de internação do recém-nascido em terapia intensiva. Logo, os extremos de idade materna estavam associados ao óbito neonatal, bem como outras condições maternas como o suo de substancias psicoativas e diagnóstico prévio de infecções sexualmente transmissíveis ${ }^{(13)}$.

A maioria das mães dos RN admitidos na UTI neonatal vive com seus companheiros em união estável, o que constitui um fator protetor. Além disso estas mulheres realizaram mais de cinco consultas de pré-natal, cumprindo assim a recomendação do Ministério da Saúde, que orienta que sejam efetuadas, no mínimo seis consultas durante a gestação(13). Contudo, os filhos necessitaram de cuidados intensivos neonatais. Dessa forma, o serviço deve assegurar a qualificação desta assistência, e suscita a importância de melhoria nos serviços de em atenção pré-natal, parto e nascimento ${ }^{(12)}$.

Em relação ao tipo de gestação, a maioria das mães de bebês internados na UTI neonatal tiveram gestação única, porém foram partos do tipo pré-termo (que aconteceram até 36 semanas de gestação).A gravidez múltipla apresenta maior risco para prematuridade e baixo peso. Neste sentido, um estudo realizado no Sul do País analisando os fatores associados à mortalidade no período neonatal em gestantes de alto risco evidenciou que as condições de óbito em recém-nascidos no período neonatal estão associadas à prematuridade, peso ao nascer e Índice de Apgar, além da malformação fetal e gestação múltipla ${ }^{(14)}$.

Com relação ao parto, houve um percentual de cesáreas que se igualou ao de partos normais. Isto está de acordo com a elevação das taxas de cesáreas no Brasil, que em 2016, foi o segundo país com mais cesáreas no mundo ${ }^{(15)}$. Neste mesmo ano, o MS aprovou as "Diretrizes de Atenção à Gestante: a operação cesariana" que define uma taxa ótima de cesáreas ajustada para o Brasil, por meio de instrumento da OMS, entre $25 \%$ e $30 \%(16)$.

O percentual de partos cesáreos verificados neste estudo chama a atenção e instiga recomendações a cerca da instrução e empoderamento das mulheres quanto a escolha do tipo de parto, e sobre a importância de reduzir intervenções médicas desnecessárias ${ }^{(17)}$. Além disso, sabe-se que a via vaginal contribui para a saúde neonatal, o fortalecimento do sistema imunológico, desenvolvimento do ritmo cardíaco e a maturação pulmonar, enquanto a cesárea oferece maior risco para a prematuridade e baixo peso ao nascer ${ }^{(18)}$.

A maioria dos RN eram do sexo masculino e de cor parda e peso ao nascer de 2.500 gramas ou mais. Entretanto, chama a atenção o percentual de BPN, fator de risco para morbimortalidade neonatal devido a imaturidade fisiológica que envolvem alterações respiratórias, metabólicas e imunológicas de grave repercussão. Como consequência, eleva-se o risco de internação em UTI neonatal e de desfechos negativos, tais como, complicações infecciosas e óbito(II).

Quanto ao índice de Apgar, verificou-se que no $1^{\circ} \mathrm{mi}-$ nuto de vida, os RNs estudados obtiveram, em sua maioria, o índice de 4 a 7, o que indica que não tiveram boa vitalidade ao nascer. No $5^{\circ}$ minuto, apesar da maioria dos bebês terem apresentado boa vitalidade, um número expressivo deles apresentou Apgar entre 4 e 7. Estudo com amostra semelhante obteve o escore de Apgar (menor que 7) com grau de asfixia moderada no $1^{\circ}$ minuto e no $5^{\circ}$ minuto, 0 qual se mostrou associado ao óbito neonatal $\left.\right|^{(14)}$. 
A vitalidade ao nascer é um processo essencial a adaptação do RN ao ambiente extrauterino, até atingir a estabilidade. Quando os procedimentos realizados são ineficazes, aumentam as possibilidades de internações na UTI neonatal, sendo o Apgar menor que 7 no $1^{\circ}$ e $5^{\circ}$ minuto considerado um fator de risco para a mortalidade neonatal. Neste sentido, estudiosos atribuem a baixa vitalidade a qualidade da assistência prestada ao parto e ao recém-nascido na sala de parto(19).

Sobre as demandas de cuidados, um terço dos RNs estudados necessitou de reanimação cardiopulmonar (RCP) indicando que tiveram asfixia ao nascer. Este achado pode estar relacionado ao quantitativo de partos pré-termos, que resultam na imaturidade pulmonar e necessidade de suporte ventilatório. Do mesmo modo, em outros estudos os RNs recebem este suporte com frequência caracterizado por oxigenoterapia e ventilação mecânica ${ }^{(11)}$.

Destaca-se crescimento da tendência ao uso de estratégias ventilatórias menos agressivas ao $\mathrm{RN}$, a utilização de novos modos ventilatórios, a retirada precoce da ventilação invasiva ou o uso da ventilação não invasiva mesmo naqueles $\mathrm{RN}$ mais prematuros e de baixo peso(II).

Corroborando com tais achados, as principais causas de internação dos RN na UTI neonatal, foram a Síndrome do desconforto respiratório (SDR), seguida pela prematuridade. Resultados semelhantes foram encontrados na literatura ${ }^{(20)}$. Sendo um dos fatores de risco para SDR a própria imaturidade pulmonar, pela deficiência de surfactante alveolar ${ }^{(13)}$. Isto torna coerente que no presente estudo, a maioria dos RNs serem pré-termos e sofreram de desconforto respiratório.

A prematuridade tem grande associação com a mortalidade neonatal, ou seja, quanto menor a idade gestacional do RN, maior é o risco de óbito, o que remete à preocupação em relação às taxas de nascimento pré-termos. Em 2010, mais de uma em cada 10 crianças nascidas vivas foram prematuras, correspondendo a aproximadamente 15 milhões de nascimentos prematuros, dos quais mais de um milhão morreram por essa causa. A prematuridade é, portanto, a principal causa de morbidade e mortalidade neonatal constituindo-se em um grande desafio para a saúde pública ${ }^{(14)}$.

Neste cenário, faz-se necessário a determinação das causas da prematuridade, com especial atenção ao pré-natal de risco, pois através do acesso aos serviços de saúde, com a realização de pré-natal mínimo, vacinação, exames de rotina, diagnóstico, as intervenções poderão ser realizadas para que haja diminuição da ocorrência de partos prematuros e, consequentemente, redução da taxa de mortalidade infantil(21). Por outro lado, o aumento da prematuridade deste estudo pode ser explicado pelo número expressivo de mães com mais de 35 anos de idade, período tardio para gestar e com risco de complicações.

Em relação ao tempo de permanência do neonato na UTI neonatal, observou-se que os RNs permaneciam menos de um mês na unidade, mas a realidade pode ser divergente ${ }^{(20)}$. No entanto, é necessário considerar que os RNs se tornam mais propensos a riscos infecciosos quando a internação é prolongada. Contudo, a maioria das mortes relacionadas com a sepse neonatal (SN) é evitável se esta for diagnosticada precocemente e tratada com terapia antimicrobiana apropriada ${ }^{(17)}$.

Quanto aos desfechos clínicos dos recém-nascidos internados na UTI em estudo, observou-se que a maioria deles evoluíram para a alta, mas é válido destacar o número de óbitos nesta amostra. Outros estudos que investigaram esta variável em UTI Neonatais, encontraram resultados com valores diversos referentes à alta $\mathrm{e}$ óbito, porém, todos com maiores percentuais de altas ${ }^{(11)}$.

Ainda sobre o desfecho dos recém-nascidos, percebeu-se uma pequena demanda dessa população para o centro de referência na capital do estado. Este dado aponta para a descentralização dos serviços de saúde, um dos princípios do Sistema Único de Saúde (SUS). E ainda demonstra eficiência do serviço estudado, visto que os RN admitidos apresentavam demandas de cuidados especializados e críticos. Vale ressaltar que o tempo de transporte desses $\mathrm{RN}$ dos municípios para a capital proporciona menos chances de sobrevida em decorrência do tempo gasto no deslocamento ${ }^{(16)}$.

Contudo, são pontos fortes do estudo: (I) ○ levantamento de características, tanto das mães como dos RNs, que irão auxiliar os profissionais de saúde da UTI neonatal estudada no direcionamento de ações em prol da saúde dos neonatos, considerando a recente implantação do serviço; (2) o estudo poderá subsidiar o planejamento de uma assistência de qualidade durante a gestação, atentando para o empoderamento das mulheres em relação a escolha do tipo de parto e necessidade de evitar cesárea programada desnecessária; e (3) os resultados obtidos representam condições de alerta para o monitoramento da morbimortalidade neonatal no município e podem ser utilizados para avaliar a qualidade dos serviços de saúde.

Destaca-se como limitação o mau preenchimento de alguns prontuários dos $\mathrm{RN}$, o que dificultou a coleta dos dados. Quanto a isso, é preciso qualificar o profissional de saúde para a utilização destas tecnologias da informação, visto que isso influencia na fidedignidade dos dados e consequentemente, a confiabilidade dos estudos que lidam com tais fontes.

\section{CONCLUSÃO}

Os achados deste estudo fortalecem a literatura científica relacionada e apontam para um grupo de mães do- 
nas de casa em idade fértil, ensino médio completo, união estável. O parto ocorreu pré-termo, em sua maioria, ainda que as gestantes tenham cumprindo com as recomendações do Ministério da Saúde em realizarem mais de 5 consultas de pré-natal. Os números de cesáreas e partos vaginais foram equiparados.

Os RNs apresentaram como características: sexo masculino, baixa vitalidade ao nascer, condições de prematuridade e síndrome do desconforto respiratório, características importantes para o desfecho clínico e que demandam assistência em saúde qualificada.

Os resultados direcionam para a necessidade do preparo da equipe na prevenção dos agravos destacados, e isto envolve a qualidade de saúde e de vida da população. Compreende-se, portanto, a necessidade de se traçar ações na saúde pública e privada com foco no trabalho pré-natal, identificando precocemente os sinais de risco ou de alteração durante a gestação.

Assim, os objetivos do estudo foram alcançados, pois foi possível identificar fatores que colaboraram para que o RN necessitasse de um leito de UTI neonatal, dentre eles o desconforto respiratório e a prematuridade. Dessa forma, recomenda-se outros estudos analíticos que esclareçam fatores associados a internação em UTI neonatal e que possam auxiliar na redução dos agravos e óbitos neonatais no Brasil. 


\section{REFERÊNCIAS}

I. Damian A, Waterkemper R, Paludo AC. Perfil de neonatos internados em unidade de tratamento intensivo neonatal: estudo transversal. Arquivos de Ciências da Saúde [internet] 2016 . [acesso em 19 de outubro de 20।8]; 23 (2): I00105. Disponível em: <http://www.cienciasdasaude.famerp. br/index.php/racs/article/view/308>.

2. Gaiva MAM, Fujimori E, Sato APS. Maternal and child risk factors associated with neonatal mortality. Texto \& Contexto-Enfermagem [internet] 2016. [cited 2018 apr 2]; 25 (4): e22900I5. Available from:<http://www.scielo.br/scielo.php?script=sci_arttext\&pi$d=S 0$ | 04-070720 | 60004003 | $8 \&$ lng=en>.

3. Fialho FA, Dias IMAV, Silva LR, Santos RS, Salvador M.Tecnologias aplicadas pela enfermagem no cuidado neonatal. Revista Baiana Enfermagem [internet] 2015. [acesso em 19 de outubro de 20I8]; 29(I):23-32. Disponível em:<https:// rigs.ufba.br/index.php/enfermagem/article/view//2309>. Acesso em 05 de maio. 2018.

4. ONU. Organização das Nações Unidas. Assembleia Geral das Nações Unidas 7 mil recém-nascido morrem por dia no mundo [internet]. Nova lorque: ONU; 2017 [acesso em 23 mar 2019].Disponível em: <https://nacoesunidas.org/ onu-7-mil-recem-nascidos-morrem-por-dia-no-mundo/>.

5. Mendonça JG, Guimarães MJB, Mendonça VM, Portugal JL, Mendonça CG.Perfil das internações em Unidades de Terapia Intensiva Pediátrica do Sistema Único de Saúde no estado de Pernambuco, Brasil. Ciência \& Saúde Coletiva [internet] 2019. [acesso em 4 de abril de 2020]; 24(3):907-916. Disponível em:<http://www.scielo.br/scielo.php?script=sci_abstract\&pi$\mathrm{d}=\mathrm{S}|4| 3-8|2320| 9000300907 \& \mid \mathrm{ng}=$ en\&nrm=iso\&tlng=pt $>$.

6. Gonzaga ICA, Santos SLD, Silva NRV, Campelo V.Atenção prénatal e fatores de risco associados à prematuridade e baixo peso ao nascer em capital do nordeste brasileiro. Ciência \& Saúde Coletiva [internet] 2016.[acesso em 10 de maio de 2019];2 (6): I965- I974. Disponível em:<https://www.scielosp. org/scielo.php?.pid=S|4|3-8| $2320 \mid 600060$ | 965\&script=sci_ arttext\&tlng=pt $>$.

7. Mucha F, Franco SC, Silva GAG. The frequency and characteristics of mothers and newborns associated with the admission of neonates to ICU in the municipality of Joinville, Santa Catarina. Rev. Bras. de Saúde Mat. Infantil [internet] 20I5. [acesso em 8 de maio de 2019]; I5(2): 201-208. Disponível em:<file://localhost/<http/::www.scielo.br:pdf:rbsmi:v|5n2: I 1 9-3829-rbsmi-|5-02-0201.pdf>.

8. Brasil.Atenção Básica:Atenção ao pré-natal de baixo risco. Caderno n. 32. Ministério da Saúde. Secretaria de Atenção à Saúde. Departamento de Atenção Básica [internet]. Brasília: Ministério da Saúde, 2012 [acesso em 25 de maio de 2019]. Disponível em: <http://bvsms.saude.gov.br/bvs/publicacoes/cadernos_atencao_basica_32_prenatal.pdf>.

9. Brasil. Ministério da Saúde. Resolução n 510 de 07 de abril de 2016. Dispõe sobre as normas aplicáveis a pesquisas em
Ciências Humanas e Sociais cujos procedimentos metodológicos envolvam a utilização de dados diretamente obtidos com os participantes. Brasília: Ministério da Saúde; 2016. [acesso em 24 de maio de 2018]. Disponível em: < http:// conselho.saude.gov.br/resolucoes/2016/Reso510.pdf $>$.

10. Sociedade Brasileira de Pediatria. SPB em Ação. Faltam 3,3 mil leitos de UTI neonatal no País, denuncia a SBP ao cobrar medidas para o Nascimento Seguro de brasileiros. [internet] Rio de janeiro: SPB; 2018. [acesso em 20 abril de 2019]. Disponível em: <https://www.sbp.com.br/imprensa/ detalhe/nid/faltam-33-mil-leitos-de-uti-neonatal-no-paisdenuncia-a-sbp-ao-cobrar-medidas-para-o-nascimento-seguro-de-brasileiros/>.

I I. Lima SS, Silva SM,Avila PES, Nicolau MV, Neves PFM.Aspectos clínicos de recém-nascidos admitidos em Unidade de Terapia Intensiva de hospital de referência da Região Norte do Brasil.ABCS Health Sciences [internet]. 2015. [acesso em 4 de abril de 2020]; 40 (2): 62-66. Disponível em: <https:// pesquisa.bvsalud.org/portal/resource/pt/lil-7548|6>.

I2. Mucha F, Franco SC, Silva GAG. Frequência e características maternas e do recém nascido associadas à internação de neonatos em UTI no município de Joinville, Santa Catarina - 20I2. Rev. Bras. Saude Mater. Infant. [Internet]. 2015 [acesso em 6 de abril de 2020]; I5(2): 20I-208. Disponível em:<http://www.scielo.br/scielo.php?script=sci_arttext\&pid=SI 5 | 9-382920 | 500020020 | \&Ing=en>.

13. Brasil. Ministério da Saúde. Secretaria de Atenção à Saúde. Departamento de Ações Programáticas Estratégicas. Atenção à saúde do recém-nascido : guia para os profissionais de saúde I Ministério da Saúde, Secretaria de Atenção à Saúde, Departamento de Ações Programáticas Estratégicas. 2. ed. atual. [Internet]. Brasília: Ministério da Saúde, 2014. [acesso em 6 de abril de 2020]. Disponível em:<http://bvsms.saude.gov.br/bvs/ publicacoes/atencao_saude_recem_nascido_v2.pdf>.

14. Demitto M de O, Gravena AAF, Dell'Agnolo CM, Antunes MB, Pelloso SM. Gestação de alto risco e fatores associados ao óbito neonatal. Rev. esc. enferm. USP [Internet]. 2017 [acesso em 4 de abril de 2019]; 5I: e03208. Disponível em:<http://www.scielo.br/scielo.php?script=sci_arttext\&pid=S0080-623420 I $7000100409 \&$ Ing=en>.

I5. Boerma T, Ronsmans C, Melesse DY, Barros AJD, Barros FC, Liang J, MD, Moller AB,

Say L, Hosseinpoor AR, Yi M, Rabello Neto, DL, Temmerman M. Global epidemiology of use of and disparities in caesarean sections. The Lancet [internet], 2018 [acess 2019 out 8]; 392 (1):|34|-1348. Available from:<http://dx.doi.org/10.1016/ s0 | 40-6736(18)31928-7>.

16. Brasil. Comissão Nacional de Incorporação de Tecnologias no SUS. Diretrizes de Atenção à Gestante: a operação cesariana. [internet] Brasília: Ministério da Saúde, 20I5. [acesso em 20 de maio de 2019]. Disponível:<http://conitec.gov. br/images/Relatorios/2016/Relatorio_Diretrizes-Cesariana_final.pdf>. 
17. Sousa NA, Coelho CGV, Mesquita CHS, Pires FGB, Rosa PB, Brito ILP. Sepse neonatal - perfil microbiológico e sensibilidade antimicrobiana em um hospital no Nordeste do Brasil. Revista Bras. de análises clínicas [internet]. 2019 [acesso em 6 de abril de 2020]; 5I(I): 46-5I. Disponível em: <http://www.rbac.org. br/artigos/sepse-neonatal-perfil-microbiologico-e-sensibilidade -antimicrobiana-em-um-hospital-no-nordeste-do-brasil/>.

18. Moreira KFA, Bicalho BO, Santos LCS,Amaral FMGS, Órfão, $\mathrm{NH}$, Cunha MPL. Perfil e evitabilidade de [óbito neonatal em um município da Amazônia legal. Cogitare Enferm. [internet]. Abr-jun 2017 [acesso em 08 Abril 2020]; (22) 2: e48950. Disponível em:<https://revistas.ufpr.br/cogitare/ article/view/48950>.

19. Zulian AC, Lisboa DDAJ, Schecci J, Lisboa RR. Perfil dos pacientes internados na Unidade de Terapia Intensiva Neonatal. J. Ciênc. Saúde [internet]. 2018 [acesso em: 08 Abril
2020]; I(3):9-19. Disponível em:<https://revistas.ufpi.br/index.php/rehu/article/view/83 I5>.

20. Freitas MCN, Sousa AOB, Cabral SAAO, Alencar MCB, Guedes MSSE, Oliveira GF. Caracterização dos RecémNascidos Internados em Unidades de Terapia Intensiva. Rev. Mult. Psic. [internet]. 2018 [acesso em 08 Abril 2020];12 (40):228-242. Disponível em:<https://idonline.emnuvens. com.br/id/article/view/III0>.

21. Borbolato BM, Cardoso MP. O Impacto do pré-natal na prevenção do parto prematuro. Revista Thêma et Scientia [internet]. 2015 [acesso em 09 Abril 2020]; 5 (I): I4759. Disponível em:<https://www.fag.edu.br/upload/arquivo/ |457720323.pdf>.

Recebido: 2020-04-27

Aceito: 2020-08-01 